\title{
La théranostique : une combinaison innovante pour le diagnostic et la thérapie des cancers
}

\author{
The theranostic: an innovative combination for the diagnosis and therapy of can- \\ cers
}

\author{
Nassima Mouassaoui' \\ ${ }^{1}$ Laboratoire de pharmacie galénique, Faculté de Médecine d'Oran, Université d'Oran1
}

\begin{abstract}
Article :. «Des nano-vecteurs multitâches combinant imagerie et traitement pour les cancers.»

Sanchez Clément*

La lettre du Collège de France 40 (2015): 40-41. Mis en ligne le 01 septembre 2015: http://lettre-cdf.revues.org/2105.

"Professeur à Université Pierre-et-Marie-Curie « UPMC », au Collège de France.
\end{abstract}

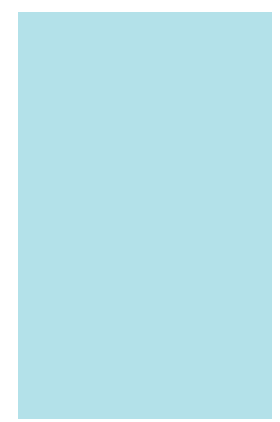

\begin{abstract}
La recherche en cancérologie doit répondre à un double défi : le diagnostic précoce des tumeurs pour permettre une thérapie efficace et le développement de nouvelles thérapies ciblées. Dans la lutte contre le cancer, les recherches actuelles s'orientent vers les nanotechnologies (nanomédicaments) qui présentent de nouvelles approches de diagnostic et de traitement : théranostique. Cet article traite de l'apport des nanotechnologies pour la conception de nouveaux médicaments destinés à la thérapeutique et au diagnostic.
\end{abstract}

Des nano-vecteurs multifonctionnels pour la théranostique: Qu'est-ce que c'est?

La théranostique, est motivée par l'emploi de nano-vecteurs permettant d'aider une substance active ou un agent de diagnostic à passer intact et en quantité maximale, les barrières biologiques qui jalonnent le parcours entre le site d'administration et la cible biologique [1]. Le but étant d'imager les tissus cancéreux et de délivrer in situ une molécule active de manière simultanée, puis de suivre l'évolution du traitement, évitant, ainsi les problèmes récurrents liés aux différences de biodistribution et de sélectivité lors de l'utilisation d'agents

Auteur correspondant : n.moussaoui@facmed-univ-oran.dz d'imagerie et de thérapie distincts [2].

Les nano-vecteurs sont définis par une taille comprise entre 5 et $300 \mathrm{~nm}$ [3], Il en existe actuellement une grande variété ayant des compositions et des structures très diverses : des nanoparticules polymériques, des nanoparticules lipidiques et des nanoparticules inorganiques d'or, d'argent ou d'oxyde de gadolinium...

Des nanoparticules d'or pour le diagnostic et la thérapie des tumeurs : quelle efficacité ?

De nombreuses expérimentations font appel aux nanoparticules d'or pour cibler et localiser des cellules avec plus de 
précision, afin de les visualiser ou de les chauffer pour les détruire. L'article du Professeur Sanchez Clément*, retrace les travaux des chercheurs de l'Imperial College de Londres et du laboratoire Chimie de la matière condensée de Paris (CNRS/ Collège de France/UPMC)a qui ont conçu et élaboré des nanoparticules hybrides d'or et de silice à base de doxorubicine de 150 nanomètres de diamètre constituées d'une coque de silice nanoporeuse abritant des clusters d'or de moins de 2 nanomètres de diamètre possédant des propriétés de : fluorescence, production de chaleur, magnétisme.

Testées sur des cellules humaines en culture et chez la souris, elles permettent de combiner :

- Deux modes de traitement des tumeurs : la chimiothérapie et la thérapie photo-thermique ;

- Trois techniques d'imagerie : Imagerie par résonance magnétique « IRM », imagerie par fluorescence et un type d'imagerie ultrasonore « photo-acoustique ».

L'incorporation d'or hydrophobe dans la sphère de silice a permis d'accroître très significativement les capacités de stockage de doxorubicine. Les chercheurs estiment que, par rapport aux vecteurs actuellement commercialisés (liposomes), la proportion des molécules qui atteindraient leur cible passerait de 5 à $95 \%$. De plus lorsqu'elles sont activées par un laser infrarouge, les particules contenant des clusters d'or émettent une fluorescence infrarouge, mais aussi suffisamment de chaleur pour détruire les cellules cancéreuses. Chez la souris, cela a permis de réduire de $55 \%$ la masse tumorale après un seul traitement.

Les scientifiques cherchent maintenant à optimiser ces na- no-vecteurs, pour un ciblage plus spécifique des cellules cancéreuses et une meilleure biodégradabilité en réduisant davantage le diamètre des clusters d'or.

\section{Conclusion}

Dans le cadre de la lutte contre le cancer, les recherches dans le domaine des nanothéranostiques faisant appel particulièrement aux nanoparticules d'or, ne cessent de susciter de l'intérêt dans l'objectif ambitieux de démontrer leur potentiel à éradiquer les tumeurs solides. Dans ce contexte, ces efforts doivent être encouragés dans la perspective d'un diagnostic précoce et précis et un traitement efficace avec moins d'effets indésirables.

\section{Notes}

a En collaboration avec des chercheurs de University College London et de Louisiana State University.

\section{Références bibliographiques}

[1] Vauthier C., Couvreur P., Nanotechnologies pour la thérapeutique et le diagnostic. Nanotech. et biotech. pour la santé. Ti597 - Techn. biomédicales. Techniques de l'ingénieur ; 2008 : 11-16.

[2] Kelkar S.S., Reineke T.M. Theranostics: combining imaging and therapy, Bioconjugate Chem. 2011, 22: 1879-1903

[3] Faraji AH, Wipf P. Nanoparticles in cellular drug delivery. Bioorg Med Chem. 2009: 2950-2962. 